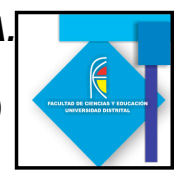

\title{
GILBERT Y LAS LÍNEAS DE FUERZA DE FARADAY: UNA RECONTEXTUALIZACIÓN HISTÓRICA PARA LA ENSEÑANZA DEL MAGNETISMO
}

\author{
GILBERT AND FARADAY'S LINES OF FORCE; A HISTORY \\ RECONTEXTUALIZATION OF THE MAGNETISM OF TEACHING
}

Magali Bravo Villamil ${ }^{1}$

\begin{abstract}
Resumen
Se pretende reconocer la importancia de la organización y construcción de experiencias sensoriales que permitan cuestionarse con el fin de dar pasó a la construcción de explicaciones con significado para el sujeto (estudiante y maestro). Para evidenciar esto en la historia de la física, se acude a la revisión de los trabajos de Gilbert y Faraday con la intención de identificar algunos rasgos del magnetismo que puedan ser llevados al aula y posibiliten discutir alrededor de las presentaciones usuales que se muestran en los libros de texto escolares. Con esta indagación se concluye que la historia de la física brinda diversas herramientas al maestro que permiten dar sentido a la física en el aula y a su vez en la labor del docente. Por otro lado y no menos importante, se mostrara que el proceso de construcción de explicaciones debe estar en continua conexión con la experiencia sensible.
\end{abstract}

Palabras clave: magnetismo, Faraday, Gilbert, experiencia sensible, enseñanza, líneas de fuerza.

\begin{abstract}
It is intended recognize the importance of organization and construction of sensorial experiences that allow questions, to make way for the construction of explanations of meaningful for the subject (students and teachers). To demonstrate this in the history of physics, it is reviewed the work of Gilbert's and Faraday's papers to identify some aspects of magnetism that can be brought into the classroom and generate discussion about common presentations are displayed in the school textbooks. With this inquiry we expect that the history of physics provides several tools that allow the teacher to make sense of physics in the classroom, as well the work of teachers. The other side and not least, I will be shown the process of construction of explanations, is in continuous connection with sensory experience,
\end{abstract}

Keywords: magnetism, Faraday, Gilbert, sensory experience, teaching, faraday's lines

\footnotetext{
${ }_{1}^{1}$ Universidad Pedagógica Nacional,Dfi592_mbravo@pedagogica.edu.co, msg19@hotmail.com
} 


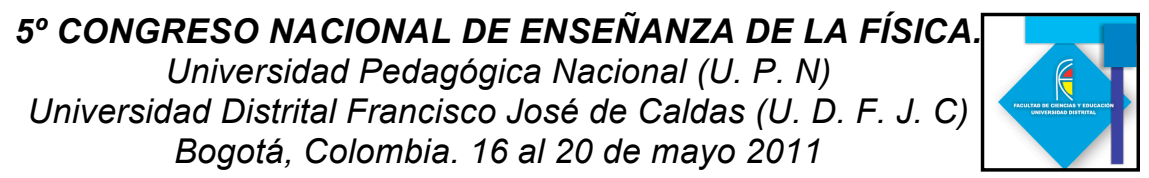

\section{Introduccion}

En la presentación usual de los libros escolares algunos aspectos del magnetismo se asumen como obvios, por ejemplo se considera que un imán tiene dos polos norte y sur, que se suelen identificar por dos colores, sin presentar algún tipo de experiencia 0 fundamento sensible que permita al estudiante asimilar de una manera lógica para su realidad este tipo de representaciones; ¿es esta presentación la más adecuada? si es así se podría inferir que el trabajo del maestro de ciencias es que los estudiantes repitan y asuman los planteamientos que los textos presentan, ahora bien, si de otra parte se asume una segunda representación de lo magnético fundamentada en el comportamiento microscópico es necesario preguntarse si cel estudiante (grado $6 \cup 11$ ) tiene alguna experiencia sensible acerca de lo microscópico?, y si itérminos como spin, moléculas magnetizadas, dominios magnéticos pueden tener algún significado para el estudiante de modo que den cuenta del comportamiento sensible de lo magnético? es pertinente preguntarse, retomando, a Gilbert si es válido explicar lo desconocido por lo más desconocido (Gilbert, 1600). Es claro que si el estudiante va a comenzar a abordar el magnetismo con este tipo explicaciones, como consecuencia, difícilmente percibirá el carácter sensible del magnetismo, por otro lado es posible darse al trabajo de revisar las elaboraciones por parte de algunos físicos con el fin de tener criterios para plantear experiencias que sean significativas para el estudiante y fortalezca la labor del docente en la enseñanza de las ciencias particularmente del magnetismo. Se recurre a la obra Gilbert De Magnete con el objetivo de reconocer en el proceso de fundamentación y desarrollo del magnetismo, proceso el cual Faraday a través de las líneas de fuerza da un gran paso en la evolución conceptual y experimental del magnetismo, esto sin dejar de lado el papel de la experiencia.

\section{El iman y los polos}

En la introducción al magnetismo la mayoría de los libros comienzan a abordar la polaridad magnética a través del campo magnético de la tierra conocido como geomagnetismo; en ellos se encuentran enunciados de este tipo:

"el polo norte geográfico de la tierra coincide con el polo norte magnético del imán y el polo sur geográfico coincide con el sur magnético del imán" (Villegas Y Ramírez, 1985) (figura 1).

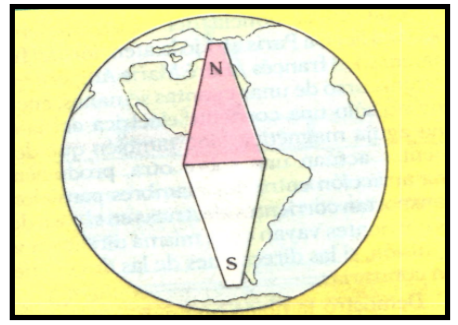

FIGURA 1. Representación del campo magnético de la tierra.

Tomada de Investiguemos 11 página 165

Con este tipo de presentaciones por un lado pareciera que la existencia de dos polos fuera algo evidente u obvio, por lo tanto, no se refleja ningún esfuerzo por caracterizar su existencia y diferenciación entre ellos. Con el fin de mostrar que al hablar de dos polos no es obvio, se plantea la siguiente pregunta ¿Cómo dar cuenta de la polaridad terrestre sin acudir a una brújula? Para tratar de dar respuesta acudo a una de las experiencias que 


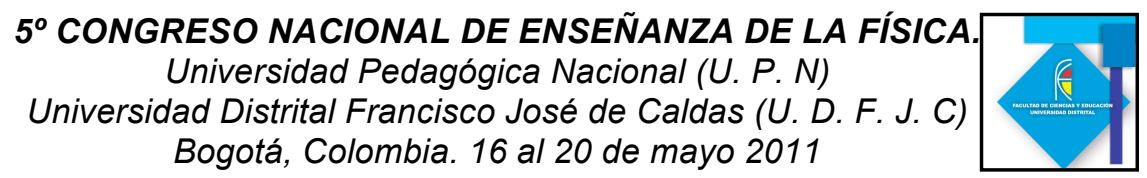

plantea Gilbert para comenzar a evidenciar la polaridad terrestre siendo esta la más sencilla:

"los polos se encuentran también en un piedra redonda (imán esférico o terella), en un versorium, una pieza de hierro tocado con un imán apoyado sobre un punto, de modo que este pueda girar libremente, en la parte superior de la piedra $A B$ se establece el versorium de tal manera que su puntero pueda permanecer en equilibrio (figura 2): marque con tiza la dirección del puntero cuando está en reposo. Después mueva el instrumento (versorium) a otro lugar y de nuevo marque la dirección en la cual el puntero mira; repita esto muchas veces en muchos puntos diferentes y encontrara, la convergencia de la dirección de las líneas, encontrar un polo en el punto $A$, el otro en el B" (Gilbert, 1600).

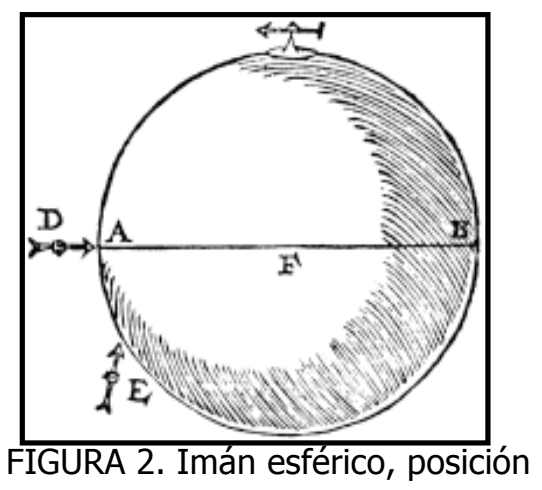

del versorium. Tomada De Magnete pagina. 25

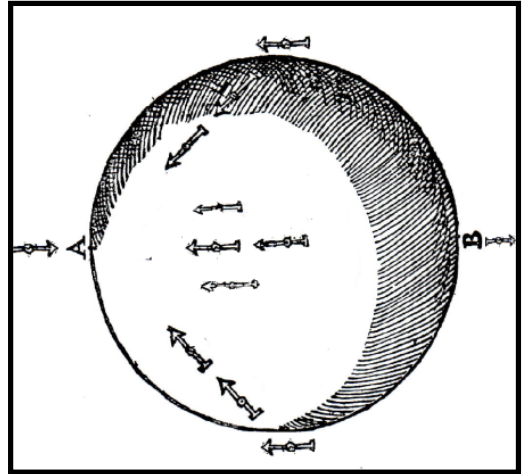

partes de la esfera. Versión modificada tomada de de magnete página 205

Si se coloca el versorium en diferentes partes de la superficie de la terella, la dirección del puntero tiende a orientarse hacia el punto $A$, sin embargo en el punto $B$ la dirección del versorium no presenta el mismo comportamiento (figura 3), esto es lo que le da paso a Gilbert para comenzar a caracterizar la polaridad de la tierra presentado con más claridad en su obra de Magnete (Gilbert, 1600).

Algunos aspectos para destacar de esta propuesta de Gilbert son: primero, la simplicidad de los instrumentos utilizados en la experiencia; la forma de la piedra o imán está justificada por la simetría de esta y su correspondencia con la forma del globo terrestre, y uso del versorium como detector de los efectos que ejerce el imán sobre el hierro. Segundo el ingenio de la experiencia y la organización de los efectos sensibles y las conclusiones a las que llega a partir de esto.

\section{Organizaciones sensibles surgimiento de las lineas de fuerza de faraday}

Para Gilbert un imán y su comportamiento es la representación que deduce el magnetismo. La experiencia muestra que esto es común encontrarlo en el diario vivir, es decir hablar de un imán y su interacción con otros objetos son elementos 


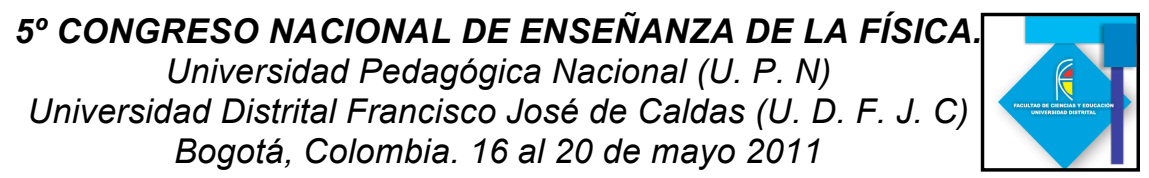

que aportan a la construcción de lo que se concibe el magnetismo; siguiendo a Gilbert la organización de diferentes experiencias dan paso a toda una

organización de lo magnético, trabajo esencial para el desarrollo de la concepción del magnetismo para Faraday.

La organización y provecho de las experiencias, es decir, darse al trabajo de plantear y deducir explicaciones que tengan sentido para el sujeto y den cuenta de los efectos sensibles detectados, se convierten en criterios que definen los cimientos de los planteamientos de Gilbert y Faraday.

Alrededor de las explicaciones de los fenómenos magnéticos (polaridad, campo) en los libros de texto escolares la palabra fuerza aparece inevitablemente, sin embargo la presentación de esta no es clara ¿Cómo caracterizar la fuerza que da cuenta de los fenómenos magnéticos? O ¿por qué no retomar la idea de fuerza de Faraday?, es importante notar como la concepción de fuerza para Faraday difiere de aquella que se considera en la mecánica según la cual fuerza es sinónimo de acción; en contraste para Faraday esta es poder o fuente de las acciones:

"una acción no es más que la manifestación de los poderes de la naturaleza. La fuerza de un huracán, la fuerza de un rio caudaloso" (Gramajo, 1993)

En una carta dirigida a Maxwell, Faraday hace la siguiente precisión sobre la manera como asume dicho término:

\footnotetext{
"percibo que no uso la palabra <<fuerza>> como usted la define <<la tendencia de un cuerpo a pasar de un lugar a otro>>. Lo que significo por la palabra es la fuente o fuentes de todas las posibles acciones de las partículas o materiales del universo; siendo estas a menudo llamadas los poderes de la naturaleza" (Faraday, 1857)
}

Para Faraday la maduración del concepto de fuerza será la base explicativa del comportamiento de la naturaleza, sin embargo si las fuerzas de la naturaleza como ya se había dicho es manifestada a través de huracanes, ríos caudalosos, y otros la concepción de fuerza que comienza a asumir debe tener una representación física, lo más real posible acorde a la capacidad de percepción humana, esta intención es uno de los aspectos que da surgimiento a las curvas magnéticas. Refiriéndose a estas Faraday aclara:

"por curvas magnéticas, yo significo las líneas de fuerza magnética, sin embargo modificada por la yuxtaposición de polos, la cual sería representada por las limaduras de hierro; o aquellas que una aguja magnética muy pequeña formarían una tangente" (Faraday, 1855) 


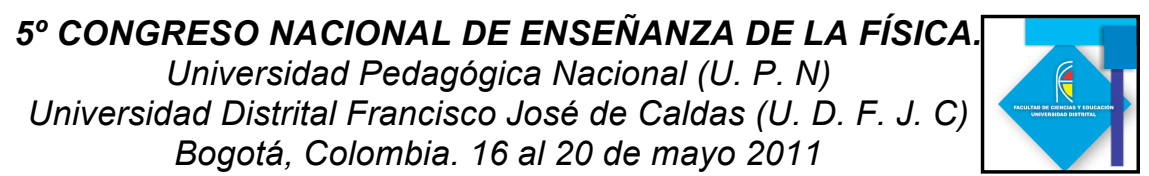

La distribución de las limaduras de hierro, o el comportamiento de la aguja magnética en presencia de un imán o de una corriente, será la representación más adecuada por tener carácter simple y consistente con la naturaleza de la fuerza.

"ahora, me parece que estas líneas se pueden emplear con gran ventaja para representar la naturaleza, condición, y cantidad comparativa de las fuerzas magnéticas y que en muchos casos ellas tienen, al menos para el razonador físico, una superioridad sobre aquel método que considera las fuerzas como concentradas en centros de acción tales como polos de los imanes"(Faraday, 1855)

A partir del planteamiento de Faraday la representación de la naturaleza de las fuerzas magnéticas y la caracterización de estas lo conllevan a concebir la existencia física de las líneas de fuerza, tangible a los sentidos y consecuente con los efectos sensibles que manifiesta el magnetismo.

Para la construcción de idea de fuerza es importante las manifestaciones y organizaciones subjetivas que son susceptibles a cambios por la experiencia humana, es decir, no se puede dar explicación a los fenómenos naturales si los planteamientos no coinciden con la experiencia sensorial, además están sujetos a modificarse; resultado de este proceso las líneas de fuerza se convierten en la mejor representación de las fuerzas magnéticas, es decir, permiten una representación simple y útil de la realidad física del fenómeno magnético.

\section{Conclusiones}

La sencillez de los planteamientos de Gilbert permiten ampliar experiencia con tanto significado que permite a su vez conceptualizarla.

La historia aporta herramientas que le permiten al maestro llevar al aula experiencias alrededor de la física que sean significativas para el estudiante.

Las experiencias significativas permiten aportar elementos al estudiante para comprender el comportamiento de situaciones sensibles.

La organización de la experiencia es reflejada en el desarrollo de la física, siendo un fundamento para la aceptación y construcción de teorías por parte de diversos físicos destacados en la historia como Faraday, Gilbert.

La recontextualización de trabajos históricos en la enseñanza han sido dejados de lado remplazándolo por los textos escolares comunes, sin embargo con este pequeño panorama se concluye que la recontextualización permite decir, concluir, preguntarse, discutir, con el fin de obtener un panorama más amplio alrededor del comportamiento de los fenómenos físicos. 


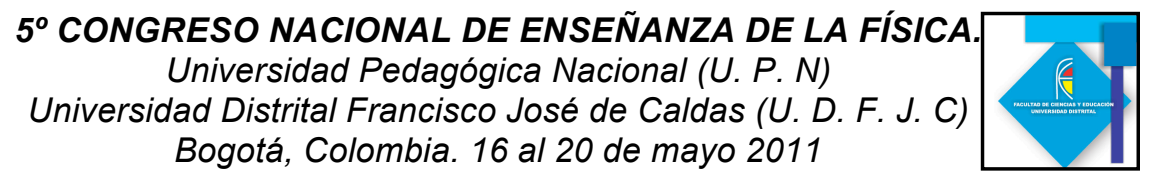

\section{Referencias Bibliográficas}

Mottelay, F.P (1893). The loadstone possesses parts differing in their natural powers, and has poles conspicuous for their properties, De Magnete (pp.22-25). Mineola, New York: Dover Publications

Gramajo, C. M. (1993). El Concepto De Carga Eléctrica En Una Concepción Clásica Campos. Las Propuestas De Michael Faraday, James Clerck Maxwell Y Heinrich Hertz. Bogotá: Universidad Pedagógica Nacional.

Faraday, M. (1855). Experimental researches in electricity. London: Bernard Quarich.

Maxwell, J.C. (1990). The scientific letters and papers of james Clerck Maxwell. Cambridge: Cambridge University Press. 